\section{Robust Stability and Performance Via Fixed-Order Dynamic Compensation: The Discrete-Time Case}

Wassim M. Haddad, Hsing-Hsin Huang, and Dennis S. Bernstein

\begin{abstract}
-discrete-time feedback control-design problem involving parametric uncertainty is considered. A quadratic bound suggested by recent work on discrete-time state space $H_{\infty}$ theory is utilized in conjunction with the guaranteed cost approach to guarantee robust stability with a robust performance bound. The principal result involves sufficient conditions for characterizing robust full- and reduced-order controllers with a worst case $\mathrm{H}_{2}$ performance bound.
\end{abstract}

\section{INTRODUCTION}

One of the fundamental problems in robust control theory concerns the stability of the state space system

$$
\dot{x}(t)=(A+\Delta A) x(t)
$$

where the nominal dynamics matrix $A$ is asymptotically stable and $\Delta A$ is an uncertain perturbation belonging to a specified uncertainty set [1]-[8]. Although the literature concerning (1.1) is quite extensive, the discrete-time analog of (1.1), namely

$$
x(k+1)=(A+\Delta A) x(k)
$$

has been relatively neglected, notable exceptions being [19]-[21]. The goal of the present note is to contribute to the system of (1.2) for both robust analysis and controller synthesis by developing new sufficient conditions for robust stability and performance. The sufficient conditions developed herein are most closely related to the approach of [21] which, in turn, is related to the discrete-time state space $H_{\infty}$ theory (see [22] and references listed therein).

After developing sufficient conditions for robust stability and performance, we apply these results to robust controller synthesis. In particular, we address the problem of full- and reducedorder dynamic compensation as well as static output feedback controllers.

\section{NOTATION AND DEFINITIONS}

Note: All matrices have real entries.

$$
\begin{aligned}
& \boldsymbol{R}, \boldsymbol{R}^{r \times s}, \boldsymbol{R}^{r}, \boldsymbol{E} \quad \text { real numbers, } r \times s \text { real matrices, } \\
& I_{r},()^{T} \\
& \boldsymbol{S}^{r}, N^{r}, \boldsymbol{P}^{r} \\
& n, m, l, r, t, n_{c} ; \bar{n} \\
& x, u, y, x_{c}, \tilde{x} \\
& A, \Delta A ; B, \Delta B ; C, \Delta C \quad n \times n \text { matrices; } n \times m \text { matrices; } l \times n \\
& A_{c}, B_{c}, C_{c}, K \\
& R^{r \times 1} \text {, expected value. } \\
& r \times r \text {, identity matrix, transpose. } \\
& r \times r \text { symmetric, nonnegative-definite, } \\
& \text { positive-definite matrices. } \\
& \text { positive integers; } n+n_{c} \\
& n, m, l, n_{c}, \tilde{n} \text {-dimensional vectors } \\
& \text { matrices. } \\
& n_{c} \times n_{c} ; n_{c} \times l ; m \times n_{c} ; m \times l \text { matri- } \\
& \text { ces. } \\
& \tilde{A}, \Delta \tilde{A} \\
& {\left[\begin{array}{cc}
A & B C_{c} \\
B_{c} C & A_{c}
\end{array}\right],\left[\begin{array}{cc}
\Delta A & \Delta B C_{c} \\
B_{c} \Delta C & 0_{n_{c}}
\end{array}\right] .}
\end{aligned}
$$

Manuscript received August 11, 1991; revised January 10, 1992. This work was supported in part by the Air Force Office of Scientific Research under Grant F49620-89-C-0011 and in part by the National Science Foundation under a Research Initiation Grant ECS-9109558.

W. M. Haddad and H. H. Huang are with the Department of Mechanical and Aerospace Engineering Florida Institute of Technology Melbourne, FL 32901.

D. S. Bernstein is with the Department of Aerospace Engineering University of Michigan Ann Arbor, MI 48109.

IEEE Log Number 9204990.

$$
\begin{aligned}
& \hat{A, \Delta} \hat{A} \\
& R_{1}, R_{2} \\
& R_{12} \\
& \hat{R} \\
& w_{1}(\cdot), w_{2}(\cdot) \\
& V_{1}, V_{2} \\
& V_{12} \\
& \tilde{w}(\cdot), \bar{V} \\
& \tilde{R} \\
& D_{0}, C_{0}, E_{0} \\
& \tilde{D}, \tilde{E} \\
& A+B K C, \Delta A+\triangle B K C \text {. } \\
& n \times n, m \times m \text { state, control weighting } \\
& \text { matrices; } R_{1} \geq 0, R_{2}>0 \text {. } \\
& n \times m \text { cross weighting matrix; } R_{1}- \\
& R_{12} R_{2}^{-1} R_{12}^{T}>0 \text {. } \\
& R_{1}+R_{12} K C+C^{T} K^{T} R_{12}^{T}+ \\
& C^{T} K^{T} R_{2} K C \text {. } \\
& n, l \text {-dimensional white noise. } \\
& \text { covariance of } w_{1}(\cdot), w_{2}(\cdot) ; V_{1} \geq 0, V_{2} \\
& >0 \text {. } \\
& n \times l \text { cross covariance of } w_{1}(\cdot), w_{2}(\cdot) \text {. } \\
& \begin{array}{l}
{\left[\begin{array}{c}
w_{1}(\cdot) \\
B_{c} w_{2}(\cdot)
\end{array}\right],\left[\begin{array}{cc}
V_{1} & V_{12} B_{c}^{T} \\
B_{c} V_{12}^{T} & B_{c} V_{2} B_{c}^{T}
\end{array}\right] .} \\
{\left[\begin{array}{cc}
R_{1} & R_{12} C_{c} \\
C_{c}^{T} R_{12}^{T} & C_{c}^{T} R_{2} C_{c}
\end{array}\right] .}
\end{array} \\
& \begin{array}{l}
n \times r, t \times n, l \times r, \text { matrice } \\
{\left[\begin{array}{c}
D_{0} \\
B_{c} E_{0}
\end{array}\right],\left[\begin{array}{ll}
C_{0} & 0_{t \times n_{c}}
\end{array}\right] .}
\end{array}
\end{aligned}
$$

\section{Robust Stability and Robust Performance Problems}

Let $\mathscr{U} \subset \boldsymbol{R}^{n \times n} \times \boldsymbol{R}^{n \times m} \times \boldsymbol{R}^{l \times n}$ denote the set of uncertain perturbations $(\Delta A, \Delta B, \Delta C)$ of the nominal plant matrices $A$, $B$, and $C$.

Robust Stability Problem: For fixed $n_{c} \leq n$, determine $\left(A_{c}, B_{c}, C_{c}\right.$ ) such that the closed-loop system consisting of the $n$ th-order controlled plant

$x(k+1)=(A+\Delta A) x(k)+(B+\Delta B) u(k)$,

measurements

$$
k=1,2, \cdots,
$$

$$
y(k)=(C+\Delta C) x(k)
$$

and $n_{c}$ th-order dynamic compensator

$$
\begin{gathered}
x_{c}(k+1)=A_{c} x_{c}(k)+B_{c} y(k) \\
u(k)=C_{c} x_{c}(k)
\end{gathered}
$$

is asymptotically stable for all $(\Delta A, \Delta B, \Delta C) \in \mathscr{U}$.

Robust Performance Problem: For fixed $n_{c} \leq n$, determine ( $A_{c}, B_{c}, C_{c}$ ) such that the closed-loop system consisting of the $n$ th-order controlled plant

$x(k+1)=(A+\Delta A) x(k)+(B+\Delta B) u(k)+w_{1}(k)$,

noisy measurements

$$
k=1,2, \cdots, \quad(3.5)
$$

$$
y(k)=(C+\Delta C) x(k)+w_{2}(k)
$$

and $n_{c}$ th-order dynamic compensator (3.3), (3.4), the performance criterion

$$
\begin{array}{r}
J\left(A_{c}, B_{c}, C_{c}\right) \triangleq \sup _{(\Delta A, \Delta B, \Delta C) \in \mathscr{L}} \limsup _{k \rightarrow \infty} E\left[x^{T}(k) R_{1} x(k)\right. \\
\left.+2 x^{T}(k) R_{12} u(k)+u^{T}(k) R_{2} u(k)\right]
\end{array}
$$

is minimized.

Remark 3.1: Note that (3.7) is precisely the discrete-time L.QG criterion except for the supremum over $\mathscr{U}$ for worst-case performance.

For each controller $\left(A_{c}, B_{c}, C_{c}\right)$ and plant variation $(\Delta A$, $\Delta B, \Delta C) \in \mathscr{U}$, the undisturbed closed-loop system (3.1)-(3.4) is given by

$$
\tilde{x}(k+1)=(\tilde{A}+\Delta \tilde{A}) \tilde{x}(k), \quad k=1,2, \cdots,
$$


while the disturbed closed-loop system (3.3)-(3.6) is

$$
\tilde{x}(k+1)=(\tilde{A}+\Delta \tilde{A}) \tilde{x}(k)+\tilde{w}(k), \quad k=1,2, \cdots,
$$
where

$$
\tilde{x}(k) \triangleq\left[x^{T}(k), x_{c}^{T}(k)\right]^{T}
$$

and $\tilde{w}(\cdot)$ is discrete-time white noise with covariance $\tilde{V} \in N^{\tilde{n}}$.

\section{SUFFICIENT CONDITIONS FOR ROBUST STABILITY AND PERformanCE}

In practice, steady-state performance is only of interest when the closed-loop system (3.8) is stable over $\mathscr{U}$. The following result expresses the performance in terms of the steady state, closed loop, second-moment matrix.

Lemma 4.1: Suppose (2.8) is stable for all $(\Delta A, \Delta B, \Delta C) \in \mathscr{U}$. Then

$$
J\left(A_{c}, B_{c}, C_{c}\right)=\sup _{(\Delta A, \Delta B, \Delta C) \in \mathscr{U}} \operatorname{tr} \tilde{Q_{\Delta} \tilde{A}} \tilde{R}
$$

where

$$
\tilde{Q}_{\Delta \tilde{A}} \triangleq \lim _{k \rightarrow \infty} E\left[\tilde{x}(k) \tilde{x}^{T}(k)\right] \in N^{\bar{n}}
$$

is the unique solution to

$$
\tilde{Q}_{\Delta \tilde{A}}=(\tilde{A}+\Delta \tilde{A}) \tilde{Q}_{\Delta \tilde{A}}(\tilde{A}+\Delta \tilde{A})^{T}+\tilde{V} .
$$

We now seek upper bounds for $J\left(A_{c}, B_{c}, C_{c}\right)$. For convenience in stating the main result of this section we assumed that $I_{t}-\tilde{E} \tilde{Q} \tilde{E}^{T}$ is positive definite for all $\tilde{Q} \in \mathscr{N} \subseteq N^{\tilde{n}}$. A sufficient condition for the positive definiteness of $I_{t}-\tilde{E} \tilde{Q} \tilde{E}^{T}$ is given in Section IV. Furthermore, for notational convenience define

$$
\Sigma \triangleq\left[\begin{array}{cc}
0_{n} & 0_{n \times n_{c}} \\
0_{n_{c} \times n} & I_{n_{c}}
\end{array}\right] .
$$

Theorem 4.1: Let $\Omega: \mathscr{N} \subseteq N^{\tilde{n}} \times R^{n_{c} \times l} \times R^{m \times n_{c}} \rightarrow S^{\tilde{n}}$ be such that

$$
\begin{aligned}
& \Delta \tilde{A} \tilde{Q} \tilde{A}^{T}+\tilde{A} \tilde{Q} \Delta \tilde{A}^{T}+\Delta \tilde{A} \tilde{Q} \Delta \tilde{A}^{T} \leq \Omega\left(\tilde{Q}, B_{c}, C_{c}\right), \\
& \quad(\Delta A, \Delta B, \Delta C) \in \mathscr{U},\left(\tilde{Q}, B_{c}, C_{c}\right) \in \mathscr{N} \times R^{n_{c} \times l} \times R^{m \times n_{c}},
\end{aligned}
$$

and, for given $\left(A_{c}, B_{c}, C_{c}\right.$ ), suppose there exists $\tilde{Q} \in \mathscr{N}$ satisfying

$$
\tilde{Q}=\tilde{A} \tilde{Q} \tilde{A}^{T}+\Omega\left(\tilde{Q}, B_{c}, C_{c}\right)+\tilde{V} .
$$

Then

$$
\left(\tilde{A}+\Delta \tilde{A}, V^{1 / 2}\right) \text { is stabilizable, for all }(\Delta A, \Delta B, \Delta C) \in \mathscr{U},
$$

if and only if

$$
\tilde{A}+\Delta \tilde{A} \text { is asymptotically stable, for all }(\Delta A, \Delta B, \Delta C) \in \mathscr{U} \text {. }
$$

In this case,

$$
\tilde{Q}_{\Delta \tilde{A}} \leq \tilde{Q} \text { for all }(\Delta A, \Delta B, \Delta C) \in \mathscr{U},
$$

where $\tilde{\mathrm{Q}}_{\Delta \tilde{A}}$ satisfies (4.2), and

$$
J\left(A_{c}, B_{c}, C_{c}\right) \leq \operatorname{tr} \tilde{Q} \tilde{R} .
$$

Furthermore,

$$
J\left(A_{c}, B_{c}, C_{c}\right) \leq \mathscr{J}\left(\tilde{Q}, A_{c}, B_{c}, C_{c}\right)
$$

where

$\mathscr{J}\left(\tilde{Q}, A_{c}, B_{c}, C_{c}\right) \triangleq \operatorname{tr}\left[\tilde{Q} \tilde{R}+\Sigma \tilde{Q} \tilde{E}^{T}\left(I_{t}-\tilde{E} \tilde{Q} \tilde{E}^{T}\right)^{-1} \tilde{E} \tilde{Q} \Sigma \tilde{R}\right]$.

Proof: For all $(\Delta A, \Delta B, \Delta C) \in \mathscr{U},(4.4)$ is equivalent to

$$
\tilde{Q}=(\tilde{A}+\Delta \tilde{A}) \tilde{Q}(\tilde{A}+\Delta \tilde{A})^{T}+\Psi\left(\tilde{Q}, B_{c}, C_{c}, \Delta \tilde{A}\right)+\tilde{V},
$$

where

$\Psi\left(\tilde{Q}, B_{c}, C_{c}, \Delta \tilde{A}\right) \triangleq \Omega\left(\tilde{Q}, B_{c}, C_{c}\right)-\Delta \tilde{A} \tilde{Q} \tilde{A}^{T}$

$$
-\tilde{A} \tilde{Q} \Delta \tilde{A}^{T}-\Delta \tilde{A} \tilde{Q} \Delta \tilde{A}^{T} .
$$

Note that by (4.3), $\Psi\left(\tilde{Q}, B_{c}, C_{c}, \Delta \tilde{A}\right) \geq 0$ for all $(\Delta A, \Delta B, \Delta C)$ $\in \mathscr{U}$. Thus if the stabilizability condition (4.5) holds for all $(\Delta A, \Delta B, \Delta C) \in \mathscr{U}$, it follows from [23, theorem 3.6] that $(\tilde{A}+$ $\left.\Delta \tilde{A},\left(\tilde{V}+\Psi\left(\tilde{Q}, B_{c}, C_{c}, \Delta \tilde{A}\right)\right)^{1 / 2}\right)$ is stabilizable for all $(\Delta A, \Delta B, \Delta C) \in \mathscr{U}$. Hence [23, lemma 12.2] implies $\tilde{A}+\Delta \tilde{A}$ is asymptotically stable for all $(\Delta A, \Delta B, \Delta C) \in \mathscr{U}$. Conversely, if $\tilde{A}+\Delta \tilde{A}$ is asymptotically stable for all $(\Delta A, \Delta B, \Delta C) \in \mathscr{U}$, then (4.5) is immediate. Next, subtracting (4.2) from (4.11) yields

$$
\begin{aligned}
\tilde{Q}-\tilde{Q}_{\Delta \tilde{A}}=(\tilde{A}+\Delta \tilde{A})\left(\tilde{Q}-\tilde{Q}_{\Delta \tilde{A}}\right)(\tilde{A}+\Delta \tilde{A})^{T} & \\
& +\Psi\left(\tilde{Q}, B_{c}, C_{c}, \Delta \tilde{A}\right)
\end{aligned}
$$

or, equivalently (since $\tilde{A}+\Delta \tilde{A}$ is asymptotically stable),

$$
\tilde{Q}-\tilde{Q}_{\Delta \tilde{A}}=\sum_{i=0}^{\infty}(\tilde{A}+\Delta \tilde{A})^{i} \Psi\left(\tilde{Q}, B_{c}, C_{c}, \Delta \tilde{A}\right)(\tilde{A}+\Delta \tilde{A})^{i^{T}} \geq 0,
$$

which implies (4.7). Next, (4.7) and (4.1) yields (4.8). Finally, (4.9) is immediate since the trace of the product of two nonnegative definite matrices is nonnegative.

Remark 4.1: If $\tilde{V}$ is positive definite then the stabilizability hypotheses of Theorem 4.1 is automatically satisfied.

Remark 4.2: Theorem 4.1 can be strengthened by noting that the stabilizability assumption is, in a sense, superfluous. To see this, first note that robust stability concerns only the undistributed system (3.8) while $\tilde{V}$ involves the disturbance noise. Hence, robust stability is guaranteed by the existence of a solution $\tilde{Q} \in \mathscr{N}$ satisfying (4.4) with $\tilde{V}$ replaced by $\alpha I_{\tilde{n}}$ for some $\alpha>0$. For this replacement stabilizability is automatic (see previous remark). For robust performance, however, $\tilde{Q}$ in (4.7) must be obtained from (4.4).

Remark 4.3: The covariance bound (4.7) can also be used to analyze the effect of disturbances on specified state variables. For example, if $E_{1} \in \boldsymbol{R}^{q \times n}$, then (4.7) implies

$$
\left[\begin{array}{ll}
E_{1} & 0_{q \times n_{c}}
\end{array}\right] \tilde{Q}_{\Delta \tilde{A}}\left[\begin{array}{c}
E_{1}^{T} \\
0_{n_{c} \times q}
\end{array}\right] \leq\left[\begin{array}{ll}
E_{1} & 0_{q \times n_{c}}
\end{array}\right] \tilde{Q}\left[\begin{array}{c}
E_{1}^{T} \\
0_{n_{c} \times q}
\end{array}\right]
$$

so that the right-hand side of (4.12) serves as a bound on selected state variances. For control-design purposes we effectively set $R_{1}=E_{1}^{T} E_{1}$. Similar remarks apply to obtaining bounds on the variances of control signals.

\section{UNCERTAINTY STRUCTURE}

To obtain explicit expressions for $\left(A_{c}, B_{c}, C_{c}\right)$ we require that $\Delta B=0,(\Delta A, \Delta B, \Delta C) \in \mathscr{U}$. Hence for simplicity, we write $(\Delta A, \Delta C) \in \mathscr{U}$. The dual case $\Delta B \neq 0$ and $\Delta C=0$ is treated in 
Section $X$. Thus, $\mathscr{U}$ is assumed to be of the form

$\mathscr{U}=\left\{(\Delta A, \Delta C) \in \boldsymbol{R}^{n \times n} \times \boldsymbol{R}^{l \times n}:\right.$

$$
\left.\Delta A=D_{0} F_{0} C_{0}, \Delta C=E_{0} F_{0} C_{0}, F_{0} F_{0}^{T} \leq \bar{F}\right\}
$$

where $D_{0} \in \boldsymbol{R}^{n \times r}, C_{0} \in \boldsymbol{R}^{t \times n}$, and $E_{0} \in \boldsymbol{R}^{l \times r}$ are fixed matrices denoting the structure of the uncertainty; $\bar{F} \in N^{r}$ is given uncertainty bound; and $F_{0} \in \boldsymbol{R}^{r \times t}$ is an uncertain matrix. The closed-loop system thus has structured uncertainty of the form

where

$$
\Delta \tilde{A}=\tilde{D} F_{0} \tilde{E}
$$

$$
\tilde{D}=\left[\begin{array}{c}
D_{0} \\
B_{c} E_{0}
\end{array}\right], \quad \tilde{E}=\left[\begin{array}{ll}
C_{0} & 0_{i \times n_{c}}
\end{array}\right]
$$

\section{THE QUADRATIC BOUND}

Given $\mathscr{U}$, we now specify the bound $\Omega$ satisfying (4.3). Note that because of $\Delta B=0, \Omega$ is independent of $C_{c}$. Hence, we write $\Omega\left(\tilde{Q}, B_{c}\right)$ for $\Omega\left(\tilde{Q}, B_{c}, C_{c}\right)$.

Proposition 6.1: Assume $I_{t}-\tilde{E} \tilde{Q} \tilde{E}^{T}$ is positive definite for all $\tilde{Q} \in \mathscr{N}$. Then the function

$$
\Omega\left(\tilde{Q}, B_{c}\right) \triangleq \tilde{A} \tilde{Q} \tilde{E}^{T}\left(I_{t}-\tilde{E} \tilde{Q} \tilde{E}^{T}\right)^{-1} \tilde{E} \tilde{Q} \tilde{A}^{T}+\tilde{D} \bar{F} \tilde{D}^{T}
$$

satisfies (4.3) with $\mathscr{U}$ given by (5.1).

$$
\text { Proof: }
$$

$$
\begin{aligned}
0 \leq & {\left[\tilde{A} \tilde{Q} \tilde{E}^{T}\left(I_{t}-\tilde{E} \tilde{Q} \tilde{E}^{T}\right)^{-1 / 2}-\tilde{D} F_{0}\left(I_{t}-\tilde{E} \tilde{Q} \tilde{E}^{T}\right)^{1 / 2}\right] } \\
& \cdot\left[\tilde{A} \tilde{Q} \tilde{E}^{T}\left(I_{t}-\tilde{E} \tilde{Q} \tilde{E}\right)^{-1 / 2}-\tilde{D} F_{0}\left(I_{t}-\tilde{E} \tilde{Q} \tilde{E}^{T}\right)^{1 / 2}\right]^{T} \\
= & \tilde{A} \tilde{Q} \tilde{E}^{T}\left(I_{t}-\tilde{E} \tilde{Q} \tilde{E}^{T}\right)^{-1} \tilde{E} \tilde{Q} \tilde{A}^{T}+\tilde{D} F_{0} F_{0}^{T} \tilde{D}^{T}-\tilde{D} F_{0} \tilde{E} \tilde{Q} \tilde{A}^{T} \\
& -\tilde{A} \tilde{Q} \tilde{E}^{T} F_{0}^{T} \tilde{D}^{T}-\tilde{D} F_{0} \tilde{E} \tilde{Q} \tilde{E}^{T} F_{0}^{T} \tilde{D}^{T} \\
\leq & \tilde{A} \tilde{Q} \tilde{E}^{T}\left(I_{t}-\tilde{E} \tilde{Q} \tilde{E}^{T}\right)^{-1} \tilde{E} \tilde{Q} \tilde{A}^{T}+\tilde{D} \bar{F} \tilde{D}^{T} \\
& -\left(\Delta \tilde{A} \tilde{Q} \tilde{A}^{T}+\tilde{A} \tilde{Q} \Delta \tilde{A}^{T}+\Delta \tilde{A} \tilde{Q} \Delta \tilde{A}^{T}\right) .
\end{aligned}
$$

Remark 6.1: A sufficient condition for the positive definiteness condition in Proposition 6.1 is assured by assuming that the triple $(\tilde{A}, \tilde{D}, \tilde{E})$ is strictly discrete bounded real. See $[24$, lemma 3.2] for further details.

\section{The Auxiliary Minimization Problem}

In the spirit of [17], to optimize robust performance while guaranteeing robust stability, we consider an auxiliary minimization problem.

Auxiliary Minimization Problem: Determine $\left(\tilde{Q}, A_{c}, B_{c}, C_{c}\right)$ which minimizes

$$
\mathscr{J}\left(\tilde{Q}, A_{c}, B_{c}, C_{c}\right) \triangleq \operatorname{tr}\left[\tilde{Q} \tilde{R}+\Sigma \tilde{Q} \tilde{E}^{T}\left(I_{t}-\tilde{E} \tilde{Q} \tilde{E}^{T}\right)^{-1} \tilde{E} \tilde{Q} \Sigma \tilde{R}\right]
$$

subject to

$$
\tilde{Q} \in \mathscr{N},
$$

$\tilde{Q}=\tilde{A} \tilde{Q} \tilde{A}^{T}+\tilde{A} \tilde{Q} \tilde{E}^{T}\left(I_{t}-\tilde{E} \tilde{Q} \tilde{E}^{T}\right)^{-1} \tilde{E} \tilde{Q} \tilde{A}^{T}+\tilde{D} \bar{F} \tilde{D}^{T}+\tilde{V}$,

$$
\left(\tilde{A}+\Delta \tilde{A}, \tilde{V}^{1 / 2}\right) \text { is stabilizable }(\Delta A, \Delta C) \in \mathscr{U} .
$$

Proposition 7.1: If ( $\tilde{Q}, A_{c}, B_{c}, C_{c}$ ) satisfies (7.2)-(7.4), then $\tilde{A}+\Delta \tilde{A}$ is asymptotically stable for all $(\Delta A, \Delta C) \in \mathscr{U}$ and

$$
J\left(A_{c}, B_{c}, C_{c}\right) \leq \mathscr{A}\left(\tilde{Q}, A_{c}, B_{c}, C_{c}\right) .
$$

Proof: With $\Omega$ given by (6.1), the hypotheses of Theorem 4.1 are satisfied so that robust stability is guaranteed with performance bound (4.6).

\section{NeCESSARy CONDITIONS FOR THE AuXILIARY Minimization Problem}

Rigorous derivation of the necessary conditions for the auxiliary minimization problem requires additional technical assumptions. Specifically, in addition to (7.2), we restrict $\left(\tilde{Q}, A_{c}, B_{c}, C_{c}\right)$ to the open set

$$
\begin{aligned}
\mathscr{S} \triangleq & \left\{\left(\tilde{Q}, A_{c}, B_{c}, C_{c}\right): \tilde{Q} \in \boldsymbol{P}^{\tilde{n}}, \tilde{A}+\tilde{A} \tilde{Q} \tilde{E}^{T}\left(I_{t}-\tilde{E} \tilde{Q} \tilde{E}^{T}\right)^{-1} \tilde{E}\right. \\
& \text { is asymptotically stable and }\left(A_{c}, B_{c}, C_{c}\right) \\
& \text { is controllable and observable }\} .
\end{aligned}
$$

Furthermore, the constraint (7.4) will not be accounted for explicitly since it can be shown that the compactness of $\mathscr{U}$ implies that the set of $\left(A_{c}, B_{c}, C_{c}\right)$ satisfying (7.4) is open.

Remark 8.1: The constraint $\left(\tilde{Q}, A_{c}, B_{c}, C_{c}\right) \in \mathscr{S}$ is not required for either robust stabjility or robust performance since Proposition 7.1 shows that only (7.2)-(7.4) are needed. Rather, the set $\mathscr{S}$ constitutes sufficient conditions under which the Lagrange multiplier technique is applicable to the auxiliary minimization problem. Specifically, the condition $\bar{Q} \geq 0$ replaces (7.2) by an open set constraint, the stability of $\tilde{A}+\tilde{A} \tilde{Q} \tilde{E}^{T}\left(I_{t}-\right.$ $\left.\tilde{E} \tilde{Q} \tilde{E}^{T}\right)^{-1} \tilde{E}$ serves as a normality condition, and $\left(A_{c}, B_{c}, C_{c}\right)$ minimal is a nondegeneracy condition. See [17], [19] for further details.

For arbitrary $Q, \hat{Q}, P \in R^{n \times n}$ define the following notation:

$$
\begin{gathered}
N \triangleq C_{0}^{T}\left(I_{t}-C_{0}(Q+\hat{Q}) C_{0}^{T}\right)^{-1} C_{0}, \quad S \triangleq\left(I_{n}+\hat{Q} N\right)^{-1} \\
\hat{S}_{Q} \triangleq S^{T} N=N S, \quad \Phi \triangleq I_{n}+N Q+N \hat{Q}, \\
P_{a} \triangleq B^{T} P A \Phi^{T}+R_{12}^{T}, \quad R_{2 a} \triangleq R_{2}+B^{T} P B, \\
Q_{a} \triangleq A\left[Q+\hat{Q}+(Q+\hat{Q}) N(Q+\hat{Q})-S \hat{Q} S^{T}-S \hat{Q} N \hat{Q} S^{T}\right] \\
\cdot C^{T}+D_{0} \bar{F} E_{0}^{T}+V_{12} . \\
V_{2 a} \triangleq V_{2}+C[Q+\hat{Q}+(Q+\hat{Q}) N(Q+\hat{Q}) \\
\left.-S \hat{Q} S^{T}-S \hat{Q} N \hat{Q} S^{T}\right] C^{T}+E_{0} \bar{F} E_{0}^{T} .
\end{gathered}
$$
[17].

The following factorization lemma is need. For details, see

Lemma 8.1: Let $\hat{Q}, \hat{P} \in N^{n}$ and suppose rank $\hat{Q} \hat{P}=n_{c}$. Then there exist $n_{c} \times n G, \Gamma$ and $n_{c} \times n_{c}$ invertible $M$, unique except for a change of basis in $\boldsymbol{R}^{n_{c}}$, such that

$$
\begin{gathered}
\hat{Q} \hat{P}=G^{T} M \Gamma, \\
\Gamma G^{T}=I_{n_{c}} .
\end{gathered}
$$

Furthermore, the $n \times n$ matrices

$$
\begin{gathered}
\tau \triangleq G^{T} \Gamma, \\
\tau_{\perp} \triangleq I_{n}-\tau .
\end{gathered}
$$

are idempotent and have rank $n_{c}$ and $n-n_{c}$, respectively. 
Theorem 8.1: If $\left(\tilde{Q}, A_{c}, B_{c}, C_{c}\right) \in \mathscr{S}$ solves the auxiliary minimization problem with $\mathscr{U}$ given by (5.1) then there exist $Q, P, \hat{Q}, \hat{P} \in N^{n}$ such that

$$
\begin{gathered}
\tilde{Q}=\left[\begin{array}{cc}
Q+\hat{Q} & \hat{Q} \Gamma^{T} \\
\Gamma \hat{Q} & \Gamma \hat{Q} \Gamma^{T}
\end{array}\right], \\
A_{c}=\Gamma\left(A \Phi^{T}-B R_{2 a}^{-1} P_{a}-Q_{a} V_{2 a}^{-1} \Phi^{T}\right) S G^{T}, \\
B_{c}=\Gamma Q_{a} V_{2 a}^{-1}, \\
C_{c}=-R_{2 a}^{-1} P_{a} S G^{T},
\end{gathered}
$$

and such that $Q, P, \hat{Q}, \hat{P}$ satisfy

$$
\begin{aligned}
Q= & A Q A^{T}+A Q \hat{S}_{Q} Q A^{T}+V_{1}+D_{0} \bar{F} D_{0}^{T}-Q_{a} V_{2 a}^{-1} Q_{a}^{T} \\
& +\tau_{\perp}\left[Q_{a} V_{2 a}^{-1} Q_{a}^{T}+\left(A \Phi^{T}-B R_{2 a}^{-1} P_{a}\right)\right. \\
& \cdot S \hat{Q} S^{T}\left(A \Phi^{T}-B R_{2 a}^{-1} P_{a}\right)^{T} \\
& \left.+\left(A \Phi^{T}-B R_{2 a}^{-1} P_{a}\right) S \hat{Q} N \hat{Q} S^{T}\left(A \Phi^{T}-B R_{2 a}^{-1} P_{a}\right)^{T}\right] \tau_{\perp}^{T},
\end{aligned}
$$

$$
\begin{aligned}
P= & \Phi A^{T} P A \Phi^{T}-P_{a}^{T} R_{2 a}^{-1} P_{a}+R_{1}+\tau_{\perp}^{T} S^{T} \\
& \cdot\left[P_{a}^{T} R_{2 a}^{-1} P_{a}+\Phi\left(A-Q_{a} V_{2 a}^{-1} C\right)^{T} \hat{P}\left(A-Q_{a} V_{2 a}^{-1} C\right) \Phi^{T}\right] S \tau_{\perp},
\end{aligned}
$$

$$
\begin{gathered}
\hat{Q}=\tau\left[\left(A \Phi^{T}-B R_{2 a}^{-1} P_{a}\right) S \hat{Q} S^{T}\left(A \Phi^{T}-B R_{2 a}^{-1} P_{a}\right)^{T}+Q_{a} V_{2 a}^{-1} Q_{a}^{T}\right. \\
\left.+\left(A \Phi^{T}-B R_{2 a}^{-1} P_{a}\right) S \hat{Q} N \hat{Q} S^{T}\left(A \Phi^{T}-B R_{2 a}^{-1} P_{a}\right)^{T}\right] \tau^{T}, \quad(8.11) \\
\hat{P}=\tau^{T} S^{T}\left[\Phi\left(A-Q_{a} V_{2 a}^{-1} C\right)^{T} \hat{P}\left(A-Q_{a} V_{2 a}^{-1} C\right) \Phi^{T}\right. \\
\left.+P_{a}^{T} R_{2 a}^{-1} P_{a}\right] S \tau, \quad(8.12)
\end{gathered}
$$

$$
\operatorname{rank} \hat{Q}=\operatorname{rank} \hat{P}=\operatorname{rank} \hat{Q} \hat{P}=n_{c} .
$$

Furthermore, the auxiliary cost is given by

$$
\begin{aligned}
\mathscr{J}\left(\tilde{Q}, A_{c}, B_{c}, C_{c}\right)=\operatorname{tr}\left[Q R_{1}+\hat{Q}\right. & \left(R_{1}-2 R_{12} R_{2 a}^{-1} P_{a} S\right. \\
& \left.\left.+P_{a}^{T} R_{2 a}^{-1} R_{2} R_{2 a}^{-1} P_{a} S\right)\right] .
\end{aligned}
$$

Conversely, if there exist $Q, P, \hat{Q}, \hat{P} \in N^{n}$ satisfying (8.9)-(8.13), then $\left(\tilde{Q}, A_{c}, B_{c}, C_{c}\right)$ given by (8.5)-(8.8) satisfy (7.2) and (7.3) with cost (8.14).

Proof: The proof follows as in the proof of the discrete-time reduced-order dynamic compensation problem with multiplicative white noise given in [25]. A similar proof can also be found in [19] with the additional terms arising due to the parametric uncertainty.

Remark 8.2: Theorem 8.1 presents necessary conditions for the auxiliary minimization problem which explicitly characterize extremal quadruples $\left(\tilde{Q}, A_{c}, B_{c}, C_{c}\right)$. These necessary conditions consist of a system of two modified Riccati equations and two modified Lyapunov equations coupled by both the optimal projection $\tau$ and uncertainty terms. Several special cases can immediately be discerned. For example, in the full-order case $n_{c}=n$, set $\tau=I_{n}$ so that $\tau_{\perp}=0$. Now the last term in each of (8.9)-(8.10) can be deleted and $G$ and $\Gamma$ in (8.5)-(8.8) can be taken to be the identity. Furthermore, $\hat{P}$ plays no role so that (8.12) is superfluous. Note that in this case, (8.9) is independent of $P$. Setting further $D_{0}, E_{0}$, and $C_{0}$ to zero, it can be seen that (8.11) and (8.12) drop out, while (8.9) and (8.10) reduce to the standard separated Riccati equations of discrete-time LQG the- ory. If, alternatively, the reduced-order constraint is retained, but the uncertainty terms are deleted, then the results of [26] are recovered.

Remark 8.3: When solving (8.9)-(8.12) numerically, the uncertainty terms can be adjusted to examine tradeoffs between performance and robustness. Specifically, the bounds $\bar{F}$ and structure matrices $D_{0}, E_{0}$, and $C_{0}$ appearing in $Q_{a}$, and $V_{2 a}$, can be varied systematically to determine the region of solvability of (8.9)-(8.12).

Remark 8.4: Although (8.9)-(8.12) appear formidable, they are, in fact, quite numerically tractable. For related problems involving coupled Riccati equations, homotopic continuation methods have been shown to be effective [27], [28]. Similar algorithms for solving (8.9)-(8.12) have been developed in [29]-[31].

\section{SUFFICIENT CONDITIONS FOR RoBUST STABILITY AND PERFORMANCE}

Theorem 9.1: Suppose there exist $Q, P, \hat{Q}, \hat{P} \in N^{n}$ satisfying (8.9)-(8.13), and assume that $\left(\tilde{A}+\Delta \tilde{A}, \tilde{V}^{1 / 2}\right)$ is stabilizable for all $(\Delta A, \Delta C) \in \mathscr{U}$ with $A_{c}, B_{c}, C_{c}$ given by (8.6)-(8.8) and $\mathscr{U}$ given by (5.1). Then $\tilde{\mathrm{A}}+\Delta \tilde{\mathrm{A}}$ is asymptotically stable for all $(\Delta A, \Delta C) \in \mathscr{U}$ and the closed-loop performance is bounded by (8.14).

Proof: Theorem 8.1 implies that $\tilde{Q}$ given by (8.5) satisfies (7.2) and (7.3). With the stabilizability assumption, the result follows from Proposition 7.1.

\section{The Dual CASE}

In place of (5.1), assume now that $\Delta C=0,(\Delta A, \Delta B, \Delta C) \in$ $\mathscr{U}$, and define

$\mathscr{U}=\left\{(\Delta A, \Delta B) \in \boldsymbol{R}^{n \times n} \times \boldsymbol{R}^{n \times m}\right.$;

$$
\left.\Delta A=D_{0} F_{0} C_{0}, \Delta B=D_{0} F_{0} G_{0}, F_{0} F_{0}^{T} \leq \bar{F}\right\}
$$

where, $D_{0} \in \boldsymbol{R}^{n \times r}, C_{0} \in \boldsymbol{R}^{t \times n}$, and $G_{0} \in \boldsymbol{R}^{t \times m}$ are fixed matrices denoting the structure of the uncertainty $\bar{F}$ and $F_{0}$ are as before. For arbitrary $Q, P, \hat{P} \in \boldsymbol{R}^{n \times n}$ define the following notation:

$$
\begin{gathered}
N_{p} \triangleq D_{0}\left(I_{r}-D_{0}^{T}(P+\hat{P}) D_{0}\right)^{-1} D_{0}^{T}, \\
S_{p} \triangleq\left(I_{n}+N_{p} \hat{P}\right)^{-1}, \quad \hat{S}_{P} \triangleq S_{P}^{T} N_{P}=N_{P} S_{P}, \\
\Phi_{P} \triangleq I_{n}+N_{P} P+N_{P} \hat{P}, \quad \hat{Q}_{a} \triangleq \Phi_{P} A Q C^{T}+V_{12}, \\
\hat{V}_{2 a} \triangleq V_{2}+C Q C^{T}, \\
\hat{P}_{a} \triangleq B^{T}\left[P+\hat{P}+(P+\hat{P}) N_{P}(P+\hat{P})-S_{P}^{T} \hat{P} S_{P}-S_{P}^{T} \hat{P} N_{P} \hat{P} S_{p}\right] \\
\cdot A+G_{0}^{T} \bar{F} C_{0}+R_{12}^{T}, \\
\hat{R}_{2 a} \triangleq R_{2}+B^{T}\left[P+\hat{P}+(P+\hat{P}) N_{P}(P+\hat{P})\right. \\
\left.-S_{P}^{T} \hat{P} S_{P}-S_{P}^{T} \hat{P} N_{P} \hat{P} S_{P}\right] B+G_{0}^{T} \bar{F} G_{0} .
\end{gathered}
$$

The main result guaranteeing robust stability and performance for the dual problem can now be stated.

Theorem 10.1: Suppose there exist $P, Q, \hat{P}, \hat{Q} \in N^{n}$ satisfying (8.12) and

$$
\begin{aligned}
P= & A^{T} P A+A^{T} P S_{P} P A+R_{1}+C_{0}^{T} \bar{F} C_{0}-\hat{P}_{a}^{T} \hat{R}_{2 a}^{-1} \hat{P}_{a} \\
& +\tau_{\perp}^{T}\left[\hat{P}_{a}^{T} \hat{R}_{2 a}^{-1} \hat{P}_{a}+\left(\Phi_{P} A-\hat{Q}_{a} \hat{V}_{2 a}^{-1} C\right)^{T}\right. \\
& \cdot S_{P}^{T} \hat{P} S_{P}\left(\Phi_{P} A-\hat{Q}_{a} \hat{V}_{2 a}^{-1} C\right) \\
& \left.+\left(\Phi_{P} A-\hat{Q}_{a} \hat{V}_{2 a}^{-1} C\right)^{T} S_{P}^{T} \hat{P} N_{P} \hat{P} S_{P}\left(\Phi_{P} A-\hat{Q}_{a} \hat{V}_{2 a}^{-1} C\right)\right] \tau_{\perp}
\end{aligned}
$$




$$
\begin{gathered}
Q=\Phi_{P} A Q A^{T} \Phi_{P}^{T}-\hat{Q}_{a} \hat{V}_{2 a}^{-1} \hat{Q}_{a}^{T}+V_{1}+\tau_{\perp} S_{P}\left[\hat{Q}_{a} \hat{V}_{2 a}^{-1} \hat{Q}_{a}^{T}\right. \\
\left.+\Phi_{P}\left(A-B \hat{R}_{2 a}^{-1} \hat{P}_{a}\right) \hat{Q}\left(A-B \hat{R}_{2 a}^{-1} \hat{P}_{a}\right)^{T} \Phi_{P}^{T}\right] S_{P}^{T} \tau_{\perp}^{T}, \\
\hat{P}=\tau^{T}\left[\left(\Phi_{P} A-\hat{Q}_{a} \hat{V}_{2 a}^{-1} C\right)^{T} S_{P}^{T} \hat{P} S_{P}\left(\Phi_{P} A-\hat{Q}_{a} \hat{V}_{2 a}^{-1} C\right)\right. \\
+\hat{P}_{a}^{T} \hat{R}_{2 a}^{-1} \hat{P}_{a}+\left(\Phi_{P} A-\hat{Q}_{a} \hat{V}_{2 a}^{-1} C\right)^{T} \\
\left.\quad S_{P}^{T} \hat{P} N_{P} \hat{P} S_{P}\left(\Phi_{P} A-\hat{Q}_{a} \hat{V}_{2 a}^{-1} C\right)\right] \tau \\
\hat{Q}=\tau S_{P}\left[\Phi_{P}\left(A-B \hat{R}_{2 a}^{-1} \hat{P}_{a}\right) \hat{Q}\left(A-B \hat{R}_{2 a}^{-1} P_{a}\right)^{T} \Phi_{P}^{T}\right. \\
\left.+\hat{Q}_{a} \hat{V}_{2 a}^{-1} \hat{Q}_{a}^{T}\right] S_{P}^{T} \tau^{T},
\end{gathered}
$$

and let $A_{c}, B_{c}, C_{c}$ be given by

$$
\begin{gathered}
A_{c}=\Gamma S_{P}\left(\Phi_{P} A-\Phi_{P} B \hat{R}_{2 a}^{-1} \hat{P}_{a}-\hat{Q}_{a} \hat{V}_{2 a}^{-1} C\right) G^{T}, \\
B_{c}=\Gamma S_{P} \hat{Q}_{a} \hat{V}_{2 a}^{-1} \\
C_{c}=-\hat{R}_{2 a}^{-1} \hat{P}_{a} G^{T} .
\end{gathered}
$$

Then, $\left(\tilde{A}+\Delta \tilde{A}, \tilde{R}^{1 / 2}\right)$ is detectable for all $(\Delta A, \Delta B) \in \mathscr{U}$ if and only if $\tilde{A}+\Delta \tilde{A}$ is asymptotically stable for all for all $(\Delta A, \Delta B) \in \mathscr{U}$. In this case, the performance of the closed-loop system satisfies

$$
\begin{aligned}
J\left(A_{c}, B_{c}, C_{c}\right) \leq \operatorname{tr}\left[P V_{1}+\hat{P}\left(V_{1}\right.\right. & -2 V_{12} \hat{V}_{2 a}^{-1} \hat{Q}_{a}^{T} S_{P}^{T} \\
& \left.\left.+\hat{Q}_{a} \hat{V}_{2 a}^{-1} V_{2} \hat{V}_{2 a}^{-1} \hat{Q}_{a}^{T} S_{P}^{T}\right)\right] .
\end{aligned}
$$

Remark 10.1: Even in the case $\Delta B=0, \Delta C=0$, the performance bounds (8.14) and (10.9) are generally different. See [18] for further details.

\section{Robust Stability and Performance via Static OUTPUT FEEDBACK CONTROLLERS}

In this section we consider the robust stability and performance of Section III for static output feedback controllers. Specifically, we seek static output feedback controllers

$$
u(k)=K y(k)
$$

where

$$
y(k)=(C+\Delta C) x(k)
$$

such that the closed-loop system (3.1), (11.1) is asymptotically stable for all $(\Delta A, \Delta B, \Delta C) \in \mathscr{U}$ where $\mathscr{U}$ is given by (10.1). Similarly, the robust performance problem involves the determination of the output feedback gain $K \in \boldsymbol{R}^{m \times l}$ such that the closed-loop system (3.5), (11.1) minimizes (3.7) with $J\left(A_{c}, B_{c}, C_{c}\right)$ replaced by $J(K)$. As in the dynamic output feedback problem, in order to obtain an explicit expression for the static output feedback gain $K$ we require that $\Delta B=0$ or $\Delta C=0$, for all $(\Delta A, \Delta B, \Delta C) \in \mathscr{U}$. In this section we present the case for which $\Delta C=0$. For conciseness we omit the dual case. Next, we present sufficient conditions for robust stability and performance via static output feedback controllers. For convenience in stating this result define the notation

$$
\begin{gathered}
P_{a} \triangleq B^{T} P\left[A+D_{0}\left(I_{r}-D_{0}^{T} P D_{0}\right)^{-1} D_{0}^{T} P A\right]+G_{0}^{T} \bar{F} C_{0}+R_{12}^{T}, \\
R_{2 a} \triangleq R_{2}+B^{T}\left[P+P D_{0}\left(I_{r}-D_{0}^{T} P D_{0}\right)^{-1} D_{0}^{T} P\right] B+G_{0}^{T} \bar{F} G_{0}, \\
\nu \triangleq Q C^{T}\left(C Q C^{T}\right)^{-1} C, \quad \nu_{\perp} \triangleq I_{n}-\nu,
\end{gathered}
$$

for arbitrary $P, Q \in R^{n \times n}$.
Theorem 11.1: Suppose there exist $P, Q, \in N^{n}$ satisfying $C Q C^{T}>0$ and

$$
\begin{gathered}
P=A^{T} P A+A^{T} P D_{0}\left(I_{r}-D_{0}^{T} P D_{0}\right)^{-1} D_{0}^{T} P A+C_{0}^{T} \bar{F} C_{0} \\
+R_{1}-P_{a}^{T} R_{2 a}^{-1} P_{a}+\nu_{\perp}^{T} P_{a}^{T} R_{2 a}^{-1} P_{a} \nu_{\perp}, \\
Q=\left[I_{n}+D_{0}\left(I_{r}-D_{0}^{T} P D_{0}\right)^{-1} D_{0}^{T} P\right]\left(A-B R_{2 a}^{-1} P_{a} \nu\right) \\
\cdot Q\left(A-B R_{2 a}^{-1} P_{a} \nu\right)^{T}\left[I_{n}+D_{0}\left(I_{r}-D_{0}^{T} P D_{0}\right)^{-1} D_{0}^{T} P\right]^{T}+V,
\end{gathered}
$$

and let $K$ be given by

$$
K=-R_{2 a}^{-1} P_{a} Q C^{T}\left(C Q C^{T}\right)^{-1} .
$$

Then, $\left(\hat{A}+\Delta \hat{A}, \hat{R}^{1 / 2}\right)$ is detectable for all $(\Delta A, \Delta B) \in \mathscr{Z}$ if and only if $\hat{A}+\Delta \hat{A}$ is asymptotically stable for all $(\Delta A, \Delta B) \in$ $\mathscr{U}$. In this case, the performance of the closed-loop system satisfies

$$
J(K) \leq \operatorname{tr} P V .
$$

Remark 11.1: Several special cases can be recovered from Theorem 11.1. For example, when the full state is available, that is, $C=I_{n}$, the projection $\nu=I_{n}$ so that $\nu_{\perp}=0$. In this case (11.5) becomes

$$
K=-R_{2 a}^{-1} P_{a}
$$

and (11.3) and (11.4) specialize to

$$
\begin{aligned}
P=A^{T} P A+A^{T} P D_{0}\left(I_{r}-\right. & \left.D_{0}^{T} P D_{0}\right)^{-1} D_{0}^{T} P A \\
& +C_{0}^{T} \bar{F} C_{0}+R_{1}-P_{a}^{T} R_{2 a}^{-1} P_{a},
\end{aligned}
$$

with performance

$$
J(K) \leq \operatorname{tr} P V .
$$

This corresponds to results obtained in [21] without the performance bound (11.9). Finally, to recover the standard LQR result let $D_{0}, E_{0}, G_{0}=0$ so that (11.8) corresponds to the standard discrete regulator Riccati equation.

\section{Alternative $\Omega$-Bounds: The Linear Bound}

Since the ordering induced by the cone of nonnegative-definite matrices is only a partial ordering, it should not be expected that there exists an operator $\Omega(\cdot)$ satisfying (4.3), which is a least upper bound. Indeed, there are alternative definitions for the bound $\Omega(\cdot)$. In this section, we present a linear bound which corresponds to the discrete analog of the continuous linear bound of [18]. This bound was first reported in [19]. Specifically, the uncertainty set $\mathscr{U}$ is now assumed to be of the form

$$
\begin{array}{r}
\mathscr{U}=\left\{(\Delta A, \Delta B, \Delta C): \Delta A=\sum_{i=1}^{p} \sigma_{i} A_{i}, \Delta B=\sum_{i=1}^{p} \sigma_{i} B_{i},\right. \\
\left.\Delta C=\sum_{i=1}^{p} \sigma_{i} C_{i}, \sum_{i=1}^{p} \sigma_{i}^{2} / \alpha_{i}^{2} \leq 1,\left|\sigma_{i} \sigma_{j}\right| \leq \alpha_{i} \alpha_{j}\right\},
\end{array}
$$

where for $i=1, \cdots, p: A_{i} \in R^{n \times n}, B_{i} \in R^{n \times m}$, and $C_{i} \in R^{l \times n}$ are fixed matrices denoting the structure of the parametric uncertainty; $\alpha_{i}$ is a given positive number; and $\sigma_{i}$ is an uncertain real parameter. Note that the uncertain parameters $\sigma_{i}$ are assumed to lie in a specified ellipsoidal region in $\boldsymbol{R}^{p}$. The closed-loop system (3.8) thus has structured uncertainty of the form

$$
\Delta \tilde{A}=\sum_{i=1}^{p} \sigma_{i} \tilde{A_{i}}
$$


where

$$
\tilde{A_{i}} \triangleq\left[\begin{array}{cc}
A_{i} & B_{i} C_{c} \\
B_{c} C_{i} & 0_{n_{c}}
\end{array}\right], \quad i=1, \cdots, p .
$$

For the structure of $\mathscr{U}$ as specified by (12.1), the bound $\Omega$ satisfying (4.3) can now be given a concrete form.

Proposition 12.1: Let $\alpha$ and $\alpha_{\mathrm{ij}}$ be arbitrary positive scalars. Then the function

$$
\begin{aligned}
\Omega\left(\tilde{Q}, B_{c}, C_{c}\right) \triangleq \alpha \tilde{A} \tilde{Q} \tilde{A}^{T}+\left(1+\alpha^{-1}\right) \sum_{i=1}^{p} \alpha_{i}^{2} \tilde{A_{i}} \tilde{Q} \tilde{A}_{i}^{T} \\
+\sum_{i \leq j} \alpha_{i} \alpha_{j}\left(\alpha_{i j} \tilde{A_{i}} \tilde{Q} \tilde{A}_{i}^{T}-\alpha_{i j}^{-1} \tilde{A_{j}} \tilde{Q} \tilde{A}_{j}^{T}\right)
\end{aligned}
$$

satisfies (4.3) with $\mathscr{U}$ given by (12.1).

Proof: Note that

$$
\begin{aligned}
(\tilde{A}+ & \left.\sum_{i=1}^{p} \sigma_{i} \tilde{A_{i}}\right) \tilde{Q}\left(\tilde{A}+\sum_{i=1}^{p} \sigma_{i} \tilde{A}_{i}\right)^{T}=\tilde{A} \tilde{Q} \tilde{A}^{T}+\sum_{i=1}^{p} \sigma_{i}^{2} \tilde{A_{i}} \tilde{Q} \tilde{A}_{i}^{T} \\
& +\sum_{i=1}^{p} \sigma_{i}\left(\tilde{A} \tilde{Q} \tilde{A}_{i}^{T}+\tilde{A_{i}} \tilde{Q} \tilde{A}^{T}\right)+\sum_{i \leq j} \sigma_{i} \sigma_{j}\left(\tilde{A_{i}} \tilde{Q} \tilde{A}_{j}^{T}+\tilde{A_{j}} \tilde{Q} \tilde{A}_{i}^{T}\right)
\end{aligned}
$$

Next, the first and second terms in the right-hand side (RHS) of (12.4) are automatically bounded by $\tilde{A} \tilde{Q} \tilde{A}^{T}+\sum_{i=1}^{p} \alpha_{i}^{2} \tilde{A}_{i} \tilde{Q} \tilde{A}_{i}^{T}$. To bound the third term in the RHS of (12.4) note that

$$
\begin{aligned}
0 \leq & \sum_{i=1}^{p}\left[\left(\alpha^{1 / 2} \frac{\sigma_{i}}{\alpha_{i}}\right) \tilde{A}-\left(\frac{\alpha_{i}}{\alpha^{1 / 2}}\right) \tilde{A_{i}}\right] \\
& \cdot \tilde{Q}\left[\left(\alpha^{1 / 2} \frac{\sigma_{i}}{\alpha_{i}}\right) \tilde{A}-\left(\frac{\alpha_{i}}{\alpha^{1 / 2}}\right) \tilde{A}_{i}\right]^{T} \\
= & \sum_{i=1}^{p}\left[\alpha \frac{\sigma_{i}^{2}}{\alpha_{i}^{2}} \tilde{A} \tilde{Q} \tilde{A}^{T}+\alpha^{-1} \alpha_{i}^{2} \tilde{A_{i}} \tilde{Q} \tilde{A}_{i}^{T}-\sigma_{i}\left(\tilde{A} \tilde{Q} \tilde{A}_{i}^{T}+\tilde{A}_{i} \tilde{Q} \tilde{A}^{T}\right)\right]
\end{aligned}
$$

which, since $\sum_{i=1}^{p} \sigma_{i}^{2} / \alpha_{i}^{2} \leq 1$, implies

$$
\sum_{i=1}^{p} \sigma_{i}\left(\tilde{A} \tilde{Q} \tilde{A}_{i}^{T}+\tilde{A_{i}} \tilde{Q} \tilde{A}^{T}\right) \leq \alpha \tilde{A} \tilde{Q} \tilde{A}^{T}+\alpha^{-1} \sum_{i=1}^{p} \alpha_{i}^{2} \tilde{A_{i}} \tilde{Q} \tilde{A}_{i}^{T} .
$$

Finally, to bound the fourth term in the RHS of (12.4) note that

$$
\begin{aligned}
0 & \leq \sum_{i \leq j}\left(\alpha_{i j}^{1 / 2} \tilde{A}_{i}-\alpha_{i j}^{-1 / 2} \tilde{A}_{j}\right) \tilde{Q}\left(\alpha_{i j}^{1 / 2} \tilde{A}_{i}-\alpha_{i j}^{-1 / 2} \tilde{A}_{j}\right)^{T} \\
& =\sum_{i \leq j}\left[\alpha_{i j} \tilde{A}_{i} \tilde{Q} \tilde{A}_{i}^{T}+\alpha_{i j}^{-1} \tilde{A_{j}} \tilde{Q} \tilde{A}_{j}^{T}-\left(\tilde{A_{i}} \tilde{Q} \tilde{A}_{j}^{T}+\tilde{A_{j}} \tilde{Q} \tilde{A}_{i}^{T}\right)\right]
\end{aligned}
$$

which, since $\left|\sigma_{i} \sigma_{j}\right| \leq \alpha_{i} \alpha_{j}$, implies

$$
\begin{aligned}
& \sum_{i \leq j} \sigma_{i} \sigma_{j}\left(\tilde{A_{i}} \tilde{Q} \tilde{A}_{j}^{T}+\tilde{A_{j}} \tilde{Q} \tilde{A}_{i}^{T}\right) \\
& \leq \sum_{i \leq j} \alpha_{i} \alpha_{j}\left(\alpha_{i j} \tilde{A_{i}} \tilde{Q} \tilde{A}_{i}^{T}+\alpha_{i j}^{-1} \tilde{A_{j}} \tilde{Q} \tilde{A}_{j}^{T}\right)
\end{aligned}
$$

Combining the above bounds, shows that (12.3) satisfies (4.3) with $\mathscr{U}$ given by (12.1).

Next one can proceed as in the previous sections to derive sufficient conditions for robust stability and performance using the linear bound. For details see [19].

\section{REFERENCES}

[1] G. S. Michael and C. W. Merriam, "Stability of parametrically disturbed linear optimal control systems," J. Math. Anal. Appl. vol. 28, pp. 294-302, 1969.
[2] S. S. L. Chang and T. K. C. Peng, "Adaptive guaranteed cost control of systems with uncertain parameters," IEEE Trans. Automat. Contr., vol. AC-17, pp. 474-483, 1972.

[3] M. Eslami and D. L. Russell, "On stability with large parameter variations stemming from the direct method of Lyapunov," IEEE Trans. Automat. Contr., vol. 25, pp. 1231-1234, 1980.

[4] R. V. Patel and M. Toda, "Quantitative measures of robustness for multivariable systems," in Proc. Joint. Automat. Contr. Conf., San Francisco, CA, June 1980, paper TP8-A.

[5] E. Noldus, "Design of robust state feedback laws," Int. J. Contr., vol. 35, pp. 935-944, 1982.

[6] I. R. Petersen, "A Riccati equation approach to the design of stabilizing controllers and observers for a class of uncertain linear systems," IEEE Trans. Automat. Contr., vol. AC-30, pp. 904-907, 1985.

[7] R. K. Yedavalli, "Improved measures of stability robustness for linar state space models," IEEE Trans. Automat. Contr., vol. AC-30, pp. 577-579, 1985.

[8] C. Van Loan, "How near is a stable matrix to an unstable matrix?," Contemp. Math., vol. 47, pp. 465-478, American Mathematical Society, 1985.

[9] D. Hinrichsen and A. J. Pritchard, "Stability radii of linear systems," Syst. Contr. Lett., vol. 7, pp. 1-10, 1986.

[10] J. M. Martin and G. A. Hewer, "Smallest destabilizing perturbations for linear systems," Int. J. Contr., vol. 45, pp. 1495-1504, 1987.

[11] R. M. Biernacki, H. Hwang, and S. P. Bhattacharyya, "Robust stability with structured real parameter perturbations," IEEE Trans. Automat. Contr., vol. AC-32, pp. 495-506, 1987.

[12] K. Zhou and P. P. Khargonekar, "Stability robustness bounds for linear state-space models with structured uncertainty," IEEE Trans. Automat. Contr, vol. AC-32, pp. 621-623, 1987.

[13] D. C. Hyland and D. S. Bernstein, "The majorant Lyapunov equation: A nonnegative matrix equation for guaranteed robust stability and performance of large scale systems," IEEE Trans. Automat. Contr., vol. AC-32, pp. 1005-1013, 1987.

[14] D. S. Bernstein, "Robust output-feedback stabilization: Deterministic and stochastic perspectives," IEEE Trans. Automat. Contr., vol. AC-32, pp. 1076-1084, 1987.

[15] D. D. Siljak "Parameter space models for robust control design: A survey," IEEE Trans. Automat. Contr., vol. 34, pp. 674-688, 1989.

[16] D. S. Bernstein and W. M. Haddad, "Robust stability and performance analysis for linear dynamic systems," IEEE Trans. Automat. Contr., vol. 34, pp. 751-758, 1989.

[17] _- "Robust stability and performance via fixed-order dynamic compensation with guaranteed cost bounds," Math. Contr. Sig Syst., vol. 3, pp. 139-163, 1990.

[18] - "Robust stability and performance analysis for state space systems via quadratic Lyapunov bounds," SLAM J. Matrix Anal. Appl., vol. 11, pp. 239-271, 1990.

[19] W. M. Haddad, "Robust optimal projection control-system synthesis," Ph.D. dissertation, Dept. Mech. Eng., Florida Institute of Technology, Melbourne, FL, Mar. 1987.

[20] D. S. Bernstein and C. V. Hollot, "Robust stability for sampled-data control systems," Syst. Contr. Lett., vol. 13, pp. 217-226, 1989.

[21] K. Furuta and S. Phoojaruenchanachai, "An algebraic approach to discrete-time $H_{\infty}$ control problem," in Proc. Amer. Contr. Conf., Pittsburgh, PA, May 1990, pp. 3067-3072.

[22] W. M. Haddad, D. S. Bernstein, and D. Mustafa, "Mixed-norm $\mathrm{H}_{2} / \mathrm{H}_{\infty}$ regulation and estimation: The discrete-time case," Syst. Contr. Lett., vol. 16, pp. 235-248, 1991.

[23] W. M. Wonham, "Linear multivariable control: A geometric approach," New York: Springer-Verlag, 1979.

[24] W. M. Haddad and D. S. Bernstein, "Explicit construction of quadratic Lyapunov functions for the small gain, positivity, circle, and Popov theorems and their applications to robust stability, Part 2: Discrete-time theory," Int. J. Robust Nonlinear Contr., to ap-

[25] pear. "Optimal projection equations for discrete-time fixed-order dynamic compensation of linear systems with multiplicative white noise," Int. J. Contr., vol. 46, pp. 65-73, 1987.

[26] D. S. Berstein, L. D. Davis, and D. C. Hyland, "The optimal projection equations for reduced-order, discrete-time modeling, estimation and control," ALAA J. Guid. Contr. Dyn., vol. 9, pp. 288-293, 1986.

[27] P. T. Kabamba, R. W. Longman, and S. Jian-Guo, "A homotopy 
approach to the feedback stabilization of linear systems," $A L A A J$. Guid. Contr. Dyn., vol. 10, pp. 422-432, 1987.

[28] M. Mariton and R. Bertrand, "A homotopy algorithm for solving coupled Riccati equations," Optimiz. Contr. Appl. Methods, vol. 6, pp. 351-357, 1985.

[29] S. W. Greeley, and D. C. Hyland, "Reduced-order compensation: LQG reduction versus optimal projection using a homotopic continuation method," in Proc. 26th IEEE Conf. Decision Contr., Los Angeles, CA, Dec. 1987, pp. 742-747.

[30] S. Richter, "A homotopy algorithm for solving the optimal projection equations for fixed-order dynamic compensation: Existence, convergence and global optimality," in Proc. Amer. Contr. Conf., Minneapolis, MN, June 1987, pp. 1527-1531.

[31] S. Richter, "Reduced-order control design via the optimal projection approach: A homotopy algorithm for global optimality" in Proc. of the Sixth VPI \& SU Sym. Dyn. Contr. Large Space Structures, Blacksburg, VA, June 1987, pp. 17-30.

\section{Computations of Limit Cycles Via Higher Order Harmonic Balance Approximation}

Jorge Moiola and Guanrong Chen

\begin{abstract}
We investigate the detection of limit cycles arising from Hopf bifurcation phenomena by applying the harmonic balance method with different higher order approximations. We present the results via an enlightening graphical procedure which indicates clearly how the predictions of amplitude and frequency of a periodic solution can be improved by using higher and higher order approximations. Moreover, we provide complete and explicit formulas for the eighth-order harmonic balance approximation.
\end{abstract}

\section{INTRODUCTION}

Hopf bifurcation theory studies periodic solutions emerging from equilibrium or steady state solution when one real parameter $\mu$ of the model is varied such that a single pair of complex eigenvalues of the associated Jacobian matrix crosses the imaginary axis. Assuming that the vectorfield is smooth up to $C^{4}$ in its state variables, Hopf showed in [1] the existence of a branch of periodic solutions for $\mu<\mu_{0}$ (subcritical bifurcation) or for $\mu>\mu_{0}$ (supercritical bifurcation).

Since the appearance of this significant result, other researchers have given similar proofs using different mathematical methods ([2]-[5]). In this note, we pursue the investigations along the lines proposed originally in [4] (and later extended in [6]-[8]) to calculate the amplitudes and frequencies of limit cycles using some theory and techniques of nonlinear feedback systems. This approach has recently gained much more attention in the study of oscillations arising from the classical Hopf theorem (see, for example, [9] and [10]) as well as from some degenerate Hopf bifurcations ([11] and [12]). However, a weak point of the theory is that the results of the approximations are strictly local. To gain a better understanding of the periodic dynamic phenomena arising from Hopf bifurcation "far away" from criticality, we need to compute some additional complex

Manuscript received September 6, 1991. This work was supported in part by the RIG Program, University of Houston, Texas.

J. Moiola is with the Departamento de Ing. Eléctrica, Univ. Nacional del Sur, Avda. Alem 1253, (8000) Bahia Blanca, Argentina.

G. Chen is with the Department of Electrical Engineering, University of Houston, Houston, TX 77204-4793.

IEEE Log Number 9204991. vectors, which are obtained by applying higher order harmonic balance approximations in the feedback system configuration.

This work is an extension of the recent achievement reported in [12], to which the reader is referred for definitions of notation. The purpose of this note is twofold: i) To show the improvement in the predictions of amplitudes and frequencies of the periodic solutions when higher order harmonic balance approximations are applied, and ii) to present the final vectors after realizing an eighth-order harmonic balance between the linear plant and the memoryless nonlinear part in the feedback path. In Section II, we first outline the derivations for obtaining the explicit formulation of the vectors involved in the eighth-order harmonic balance approximation. Then, in Section III, we show an application of the new formulas and a comparison among different level higher order approximations, where the improvement of the predictions of amplitude and frequency for a periodic solution will be demonstrated by using higher and higher order harmonic balance approximations.

\section{MAIN RESULTS}

Consider a general multivariable autonomous system described by an ordinary differential equation of the form

$$
\dot{x}=A(\mu) x+B(\mu) g(C(\mu) x ; \mu)
$$

which depends on a real parameter $\mu$, together with a so-called output equation

$$
y=C(\mu) x
$$

where $A(\mu), B(\mu)$, and $C(\mu)$ are $n \times n, n \times l$, and $m \times n$ matrices, respectively, $g: R^{m} \rightarrow R^{l}$ is a nonlinear vector-valued function. By taking Laplace transforms on both sides of (1) we can separate this general nonlinear system into a dynamic linear part with a proper rational transfer function $G(s ; \mu)$ and a memoryless nonlinear part $f$, in a way similar to the classical describing function method, and write the linear part as

$$
G(s ; \mu)=C(\mu)(s I-A(\mu))^{-1} B(\mu) .
$$

Let $u=f(e, \mu)=g(y, \mu)$ and assume that $f: R^{m} \rightarrow R^{l}$ is $C^{2 q+1}$ $(q \geq 2)$ in its variable $e$. The overall feedback configuration for this setting in the frequency domain is shown in Fig. 1(a) and its justification can be found in [6].

The equilibrium solutions of (1) are defined as the values $\hat{x}$ satisfying $\dot{x}=0$. In [6] it was shown that the computation of $\hat{x}$ in the time domain is equivalent to the computation of

$$
G(0, \mu) f(\hat{e}, \mu)=-\hat{e}
$$

in the frequency domain. By linearizing the feedback path in Fig. 1(a) about the equilibrium point $\hat{e}(\mu)$, we obtain the system shown in Fig. 1(b), where $J=\left.(\partial f / \partial e)\right|_{\hat{e}}$ is called the Jacobian matrix. We can apply the generalized Nyquist stability criterion to study the stability of the steady state solutions of this linear feedback system. Moreover, by considering the graphical Hopf theorem stated in [6] and [8], we can determine the location and stability of emerging periodic solutions, that is, we can analyze for what values of $\mu$ there are limit cycles.

Consider the characteristic polynomial of the $m \times m$ openloop transfer matrix $G J$ defined by

$$
\begin{aligned}
\operatorname{det} \mid \lambda I & -G(s ; \mu) J(\mu) \mid \\
& =h(\lambda, s ; \mu) \\
& =\lambda^{t}+a_{t-1}(s ; \mu) \lambda^{t-1}+\cdots+a_{1}(s ; \mu) \lambda+a_{0}(s ; \mu) \\
& =0 ; \quad t=\min (l, m)
\end{aligned}
$$

